\title{
Diffusion kurtosis imaging and conventional diffusion weighted imaging to assess electrochemotherapy response in locally advanced pancreatic cancer
}

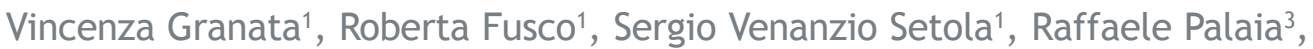 \\ Vittorio Albino ${ }^{3}$, Mauro Piccirillo ${ }^{3}$, Robert Grimm ${ }^{4}$, Antonella Petrillo ${ }^{1}$, Francesco Izzo ${ }^{3}$ \\ ${ }^{1}$ Division of Radiology, Instituto Nazionale Tumori - IRCCS - Fondazione G. Pascale, Napoli, Italia \\ 2 IGEA SpA \\ ${ }^{3}$ Division of Hepatobiliary Surgical Oncology, Unit, Instituto Nazionale Tumori, IRCCS, Fondazione G. Pascale, Napoli, Italia \\ ${ }^{4}$ Siemens Healthcare GmbH, Erlangen, Germany
}

Radiol Oncol 2019; 53(1): 15-24.

Received 24 September 2018

Accepted 18 November 2018

Correspondence to: Vincenza Granata, Division of Radiology, Instituto Nazionale Tumori - IRCCS - Fondazione G. Pascale, Napoli, Italia. Phone: +39 0815903 714; Fax:+39 0815903825; E-mail: v.granata@istitutotumori.na.it

Disclosure: The authors declare that have no conflict of interest. Roberta Fusco is an employee of IGEA S.p.A and she contributes to biomedical images processing and statistical analysis. Robert Grimm is an employee of Siemens Healthcare and develops the MR Body Diffusion Toolbox, a post-processing software to calculate IVIM and Kurtosis maps.

Background. The aim of the study was to evaluate diagnostic performance of functional parameters derived by conventional mono-exponential approach of diffusion weighted imaging (DWI) and by diffusion kurtosis imaging (DKI) in the assessment of pancreatic tumours treated with electrochemotherapy (ECT).

Patients and methods. Twenty-one consecutive patients with locally advanced pancreatic adenocarcinoma subjected to ECT were enrolled in a clinical approved trial. Among twenty-one enrolled patients, 13/21 (61.9\%) patients were subjected to MRI before and after ECT. DWI was performed with a $1.5 \mathrm{~T} \mathrm{scanner;} \mathrm{a} \mathrm{free} \mathrm{breathing} \mathrm{axial} \mathrm{single} \mathrm{shot}$ echo planar DWI pulse sequence parameters were acquired using seven b value $=0,50,100,150,400,800,1000 \mathrm{~s} /$ $\mathrm{mm}^{2}$. Apparent diffusion coefficient by conventional mono-exponential approach and mean of diffusion coefficient (MD) and mean of diffusional kurtosis (MK) by DKI approach were derived from DWI. Receiver operating characteristic (ROC) analysis was performed and sensitivity, specificity, positive and negative predictive value were calculated.

Results. Among investigated diffusion parameters, only the MD derived by DKI showed a significant variation of values between pre and post treatment ( $p=0.02$ at Wilcoxon test) and a significant statistically difference for percentage change between responders and not responders ( $p=0.01$ at Kruskal Wallis test). MD had a good diagnostic performance with a sensitivity of $80 \%$, a specificity of $100 \%$ and area under ROC of 0.933 .

Conclusions. MD derived by DKI allows identifying responders and not responders patients subject to ECT treatment. MD had higher diagnostic performance to assess ECT response compared to conventional DWI derived parameters.

Key words: pancreatic cancer; electrochemotherapy; magnetic resonance imaging; diffusion weighted imaging; diffusion kurtosis imaging

\section{Introduction}

Electrochemotherapy (ECT) is a promising tool for treatment of deep tumours. ${ }^{1-6}$ This ablative technique combines the administration of chemotherapeutic drugs with electric pulses for cell mem- brane electroporation. ${ }^{7}$ In our previous studies ${ }^{6-9}$, we demonstrated the safety and efficacy of the treatment in locally advanced pancreatic cancer. However, the correct assessment of the efficacy of ECT is a challenge for a radiologist since tumour necrosis is not always correlated with a dimension- 
al change so that the response evaluation criteria in solid tumour (RECIST) are not adequate to assess the ECT treatment. ${ }^{9}$ In this scenario, we evaluated functional parameters extracted by diffusion weighted imaging (DWI). ${ }^{10}$ The practice of DWI into a standard magnetic resonance (MR) protocol is increasing, thanks to its ability to detect and characterize a lesion so as its capability in the assessment of treatment response. ${ }^{11-12}$ Promising results for improved detection and monitoring of therapeutic effects, in terms of prediction and early response assessment, have been reported, amongst others, for the liver ${ }^{13}$, pancreas $^{14}$, kidneys ${ }^{15}$ and prostate. ${ }^{16}$ Several authors have reported that DWI sequence have a potential in the evaluation of patients with locally advanced pancreatic cancer, especially improving the staging; nevertheless, it is still unclear whether and how DWI could be helpful for identification, characterization, prognostic stratification and follow-up during treatment. DWI assessment may be done qualitatively or quantitatively, with a mono-exponential (apparent diffusion coefficient map, ADC) or bi-exponential analysis (intravoxel incoherent motion model or diffusion kurtosis imaging, DKI) of data. ${ }^{17-19}$ DWI signal is due to the water mobility that reproduces indirectly tissue structures. ${ }^{14-18}$ Traditionally DWI approach is based on the hypothesis that water molecules diffuse within a voxel following a single component direction according to a Gaussian behavior, without any restriction. ${ }^{18-19}$ However, according to the presence of microstructures, random motion or diffusion of thermally agitated water molecules within biologic tissues exhibits non-Gaussian phenomena due to the presence of microstructures. ${ }^{19}$ This model, proposed by Jensen et al. in 2005, considers a non-Gaussian diffusion model called DKI. ${ }^{19}$ This approach evaluates the kurtosis coefficient $(\mathrm{K})$ that shows the deviation of tissue diffusion from a Gaussian model, and the diffusion coefficient (D) with the correction of non-Gaussian bias. Several researches have shown that DKI is more performing than conventional ADC in tumour detecting and grading assessment. ${ }^{19-23}$ Kartalis et al. ${ }^{24}$ reported that the correction for kurtosis effects has the potential to increase the diagnostic accuracy of DWI in patients with pancreatic ductal adenocarcinoma. To the best of our knowledge, no studies that evaluated the diagnostic performance of DKI parameters to assess ECT response have been published.

The purpose of this retrospective study is to evaluate the diagnostic performance of ADC by conventional mono-exponential approach and MD and MK by DKI to assess ECT response.

\section{Patients and methods}

\section{Study population}

The patients were enrolled in a clinical phase I/II study approved by the Ethical Committee of the National Cancer Institute "G. Pascale Foundation - IRCCS" of Naples (deliberation n. 482 of 02/07/2014). The study endpoints were the feasibility and safety of ECT in the multimodal treatment of pancreatic cancer in patients with locally advanced disease and not suitable for radical surgery.

Twenty-one consecutive patients ( 11 female and 10 male) were enrolled in a clinical approved trial. Inclusion criteria were: age between 18-80 years; good mental health; life expectancy $\geq 3$ months; histologically confirmed diagnosis of pancreatic adenocarcinoma; locally advanced disease (stage III) confirmed with preoperative radiological assessment, unfit for curative surgery. Exclusion criteria were: pregnant women, significant heart disease, coagulation disturbances, allergy to bleomycin, lung and kidney dysfunction, implanted defibrillator or pacemaker, concomitant presence of distant metastases. All patient enrolled signed the informed consent. All patients enrolled with diagnosis of locally advanced pancreatic adenocarcinoma received systemic chemotherapy before ECT treatment. Two chemotherapy regimens were adopted: gemcitabine + oxaliplatin (GEMOX) or 5-FU/leucovorin, irinotecan, and oxaliplatin (FOLFIRINOX). GEMOX regime consisted of 100-minute infusion of gemcitabine at a dose 1.000 $\mathrm{mg} / \mathrm{m}^{2}$ administered on day 1 and a 2 -hour infusion of oxaliplatin at a dose of $100 \mathrm{mg} / \mathrm{m}^{2}$ administered on day 2. Treatment was repeated every 2 weeks for 3 months. FOLFIRINOX regime consisted of 2-hour intravenous infusion of oxaliplatin at a dose of $85 \mathrm{mg} / \mathrm{m}^{2}$ immediately followed 2-hour intravenous infusion of leucovorin at a dose of $400 \mathrm{mg} / \mathrm{m}^{2}$ with the addition, after 30 minutes, of 90-minute intravenous infusion of irinotecan at a dose of 180 $\mathrm{mg} / \mathrm{m}^{2}$. This treatment was immediately followed by intravenous bolus of fluorouracil at a dose of $400 \mathrm{mg} / \mathrm{m}^{2}$, followed by a continuous intravenous infusion of $2400 \mathrm{mg} / \mathrm{m}^{2}$ over a 46 -hour period every 2 weeks. Fourteen $(14 / 21,66.7 \%)$ patients were subjected to GEMOX and seven patients (7/21, 33.3\%) were injected with FOLFIRINOX before ECT treatment (median time between start of chemotherapy treatment and ECT was 124 days, range 118-139).

Among 21 enrolled patients, 13 were subjected to MRI before and after ECT. Median time between basal MR imaging assessment and ECT was 15 days (range 7-19). Median time between ECT 
TABLE 1. Patients' characteristics

\begin{tabular}{ll}
\hline Patients $(\boldsymbol{n}=\mathbf{2 1})$ & \\
\hline Histotype, \% & $100(21 / 21)$ \\
$\quad$ Adenocarcinoma & \\
Location, \% & $52.4(11 / 21)$ \\
$\quad$ Head & $47.6(10 / 21)$ \\
$\quad$ Body/tail & $5.2(2.2-9.9)$ \\
Largest diameter lesion, cm (range) & \\
Venus involvement (superior mesenteric \\
vein [SMV] or portal vein [PV]), \% \\
$\quad$ Yes & $81.0(17 / 21)$ \\
$\quad$ No & $19.0(4 / 21)$ \\
Arterial encasement, \% & \\
$\quad$ Yes & $57.1(12 / 21)$ \\
$\quad$ No & $42.9(9 / 21)$ \\
\hline
\end{tabular}

and first MR in the follow-up assessment was 38 days (range 27-72). Patient characteristics are summarized in the Table 1.

\section{ECT protocol}

ECT was performed on pancreas tumour intraoperatively, bleomycin was administrated intravenously $\left(15.000 \mathrm{IU} / \mathrm{m}^{2}\right)$ before the application of electrical pulses to the target area. Electric pulses were applied by needle electrodes with linear, hexagonal configuration or variable geometry using multiple single needles (IGEA S.p.A., Carpi, Italy) depending on the size and location of the tumours. Cliniporator $^{\mathrm{TM}}$ (IGEA S.p.A., Italy) was used to deliver electric voltage with the following parameters using linear or hexagonal geometry: 8-96 pulses of $400-730 \mathrm{~V}$ and $1000 \mathrm{~V} / \mathrm{cm}$, of $100 \mu$ s duration, at $5000 \mathrm{~Hz}$ repetition frequency. When a variable geometry was used, a single pulse of $100 \mu$ s duration at $1000 \mathrm{~V} / \mathrm{cm}$ was delivered with a single relived R-wave (ECG synchronization). Electric impulses were synchronized with the ECG for a safe delivery of the electric impulses to pancreas. ECG synchronization was done with Accusync 42 (AccuSync Medical Research Corporation, Milford, USA). Treatment was completed within the window from 8 to 40 minutes after the end of the bleomycin bolus. This time window ensures the highest concentration of drug within the lesion.

The standard used to assess treatment outcome has been the consensus between two radiological modalities (computed tomography [CT] assessed using Choi criteria ${ }^{25}$, position emission tomography-CT [PET-CT]) assessed using PERCIST criteria $^{26}$ and dynamic contrast enhanced-MRI [DCEMRI] assessed by variation in wash-in and washout slope [WIS and WOS]). Details of these criteria were reported in Granata et al. including also the specifications about the two dynamic parameters WIS and WOS extracted by DCE-MRI. ${ }^{9}$

\section{MR protocol and images analysis}

DWI was performed with a $1.5 \mathrm{~T}$ scanner (Magnetom Symphony, Siemens Medical System, Erlangen, Germany) equipped with a phased-array body coil. Patients were placed in a supine, headfirst position. A free breathing axial single shot echo planar DWI pulse sequence parameters were: repetition time $(\mathrm{TR}) /$ echo time $(\mathrm{TE})=7500 / 91 \mathrm{~ms}$; slice thickness $=3 \mathrm{~mm}$; flip angle $=90$ degrees, Matrix $=192 \times 192$ and field of view $(F O V)=340 \times$ $340 \mathrm{~mm}^{2}$; b value $=0,50,100,150,400,800,1000 \mathrm{~s} /$ $\mathrm{mm}^{2}$. Other sequences of MRI protocol were provided in the Table 2.

Regions of interest (ROIs) were manually segmented by two expert radiologists in consensus, simultaneously avoiding encircling any distortion artefacts. One radiologist with over 20 years of clinical

TABLE 2. MRI protocol parameters

\begin{tabular}{|c|c|c|c|c|c|}
\hline Sequence & Orientation & $\begin{array}{c}\mathrm{TR} / \mathrm{TE} / \mathrm{FA} \\
\text { (ms/ms/deg.) }\end{array}$ & FOV $\left(\mathrm{mm}^{2}\right)$ & $\begin{array}{l}\text { Acquisition } \\
\text { matrix }\end{array}$ & $\begin{array}{l}\text { Slice thickness/ } \\
\text { gap }(\mathrm{mm})\end{array}$ \\
\hline HASTE T2-W & Axial & $1500 / 90 / 180$ & $380 \times 380$ & $320 \times 320$ & $5 / 0$ \\
\hline FLASH T1-W, out phase & Axial & $178 / 2.3 / 80$ & $325 \times 400$ & $416 \times 412$ & $3 / 0$ \\
\hline DWI & Axial & $7500 / 91 / 90$ & $340 \times 340$ & $192 \times 192$ & $3 / 0$ \\
\hline
\end{tabular}

AT = acquisition time; DWI = diffusion-weighted imaging; FA = flip angle; FLASH = fast low angle shot; FOV = field of view; HASTE = half-Fourier acquisition single-shot turbo spinecho; TE = echo time; TR = repetition time; TWIST = time-resolved angiography with stochastic trajectories; VIBE = volumetric interpolated breath hold examination; $\mathrm{W}=$ weighted 
experience, and one with 10 years of clinical experience in interpreting abdominal MR imaging studies drew ROIs on DWI image at the highest $b$ value both on pre-treatment images and on post treatment images. The tumour was contoured slice by slice to obtain the neoplastic volume of interest (VOI). Size of VOI was variable depending by tumour size (maximum diameter range was reported in Table 1).

Features from DWI data have been computed pixel by pixel to obtain the median value of ROIs.

\section{DWI features}

Per each voxel, 3 features were extracted from DWI data using the mono-exponential approach and the DKI model.

DWI signal decay is most commonly analysed using the monoexponential model. ${ }^{17-18}$

$$
A D C=\frac{\ln \left(\frac{S_{0}}{S_{b}}\right)}{b}
$$

where $\mathrm{Sb}$ is the MRI signal intensity with diffusion weighting b, S0 is the non-diffusion-weighted signal intensity and ADC is the apparent diffusion coefficient.

Multi-b DW images were obtained by fitting of voxel-by-voxel using the diffusion kurtosis signal decay equation [2] by a two-variable linear least squares algorithm as used in previous study. ${ }^{19}$

$$
S(b)=S_{0} \exp \left(-b \cdot D+\frac{1}{6} b^{2} \cdot D^{2} \cdot K\right)
$$

In this equation, $\mathrm{D}$ is a corrected diffusion coefficient; and $\mathrm{K}$ is the excess diffusion kurtosis coefficient. $\mathrm{K}$ describes the degree that molecular motion deviates from the perfect Gaussian distribution. When $\mathrm{K}$ is equal to 0 , equation [2] evolves into a conventional monoexponential equation [1]:

The difference between $\mathrm{D}$ and ADC is that $\mathrm{D}$ is a corrected form of ADC for use in non-Gaussian circumstances.

The parameters of conventional DWI (ADC) and DKI (Mean of Diffusion Coefficient (MD) and mean of Diffusional Kurtosis (MK)) were obtained from the multi-b DWI data with all measured $b$ values using the prototype post-processing software Body Diffusion Toolbox (Siemens Healthcare, Erlangen, Germany).

\section{CT acquisition protocol and images analysis}

Non contrast-enhanced phase and triple-phase contrast-enhanced CT was performed with a 64-de- tector row scanner (Optima 660, GE Healthcare, USA). CT scanning parameters were $120 \mathrm{kVp}$, 100-470 mAs (NI 16.36), 2.5-mm slice thickness and table speed $0.984 / 1 \mathrm{~mm} /$ rotation. Scans were carried out including a region encompassing the liver from diaphragm to iliac crests. Phases were as follows; hepatic arterial phase 30-40 s after injection of $120 \mathrm{~mL}$ of a nonionic contrast medium (Iomeprol, Iomeron 400, Bracco, Milan, Italy) with a bolus-triggered technique (120 kVp; 40-60 mA; trigger threshold, $180 \mathrm{HUs}$ in descending aorta), portal and equilibrium phase $90 \mathrm{~s}$ and $120 \mathrm{~s}$ after contrast injection. The contrast medium was administered at a rate of $4 \mathrm{~mL} / \mathrm{s}$ through antecubital vein with an automated injector system (Empower CTA, E-Z-EM Inc., New York, United States). For CT images, the response to ECT was evaluated according the Choi criteria. ${ }^{25} \mathrm{CR}$ is disappearance of target lesion; PR is a decrease in tumour size $\geq 10 \%$ or decrease in tumour density $\geq 15 \%$ on CT; SD is neither PR nor PD; and PD is an increase in tumour size $\geq 10 \%$ and does not meet PR criteria by tumour density.

\section{PET/CT acquisition protocol and images analysis}

18F-FDG PET/CT studies were acquired $60 \mathrm{~min}$ after the administration of $300-385 \mathrm{MBq}$ of FDG either with a Siemens ECAT EXACT 47 or a General Electric DST 600 PET-CT scanner. Patients fasted for at least $6 \mathrm{~h}$, and blood glucose level was $<150$ $\mathrm{mg} / \mathrm{dl}$. Each patient underwent the baseline and the pre-operative study on the same scanner. Irregular volumes of interest (VOIs) were semiautomatically drawn by the expert investigator on orthogonal planes using a dedicated workstation and software. For each patient both studies were analysed at the same time in order to minimize discrepancies in VOI positioning. For each study, maximum standardized uptake value (SUVmax) values of the pancreas lesion were recorded. The analysis of 18F-FDG PET/CT results was performed by comparing measurements obtained in the pancreatic lesion at baseline (SUV1) and after treatment (SUV2). This change was expressed as the percentage of SUV reduction $(\triangle \mathrm{SUV}=(\mathrm{SUV} 1-$ SUV2)/SUV1 × 100). Objective therapeutic responses was defined according to PERCIST 1.0.26 complete metabolic response (CMR) is complete resolution of 18F-FDG uptake within the measurable target lesion and indistinguishable from surrounding background blood-pool levels with no new 18F-FDG-avid lesions; partial metabolic response 
(PMR) is reduction of a minimum of $30 \%$ in the target tumour 18F-FDG SUVmax; stable metabolic disease (SMD) is disease other than CMR, PMR, or progressive metabolic disease; and progressive metabolic disease is a $30 \%$ increase in 18F-FDG PET/CT SUVmax or advent of new 18F-FDG-avid lesions that are typical of cancer.

\section{Statistical analysis}

Continuous variables were presented as the median \pm standard deviation (SD). Wilcoxon test and the Kruskal Wallis test were performed to assess significant statistically differences between pre and post parameters value and between responders and not responders, respectively. As well, Receiver operating characteristic (ROC) curves were calculated to characterize each parameter value for evaluating the capability to differentiate responders versus non responders. The optimal cut-off values (obtained according to the maximal Youden index = sensitivity + specificity-1), the corresponding sensitivity, specificity, positive predictive value (PPV), negative predictive value (NPV) and accuracy were calculated.

A $p$ value $<0.05$ was considered statistically significant. The Statistics Toolbox of Matlab R2007a (The Math-Works Inc., Natick, MA) was used to perform statistical analysis.
TABLE 3. Electrochemotherapy (ECT) response classification for each patient

\begin{tabular}{ccccc}
\hline No. & $\begin{array}{c}\text { CT response } \\
\text { according Choi }\end{array}$ & $\begin{array}{c}\text { DCE-MRI response } \\
\text { according } \Delta \text { WIS } \\
\text { and } \Delta \text { WOS }\end{array}$ & $\begin{array}{c}\text { PET response } \\
\text { according } \\
\text { PERCIST }\end{array}$ & $\begin{array}{c}\text { Consenssus } \\
\text { among two } \\
\text { modalities }\end{array}$ \\
\hline 1 & PR & PR & PD & PR \\
2 & PR & PR & PR & PR \\
3 & PR & PR & & PR \\
4 & PR & PR & CR & PR \\
10 & PR & & PR & SD \\
11 & PR & SD & SD & SD \\
12 & SD & PR & PR & PR \\
13 & PR & SD & PR & PR \\
14 & PR & PR & SD & PR \\
17 & PR & PR & SD & PR \\
18 & PR & PR & PR & PR \\
19 & PR & PR/SD & & PR \\
20 & PR & PR & & SD \\
21 & SD & SD & & \\
\hline
\end{tabular}

Choi = Choi criteria; $\mathrm{CT}=$ computed tomography; DCE-MRI = dynamic contrast enhanced magnetic resonance imaging; PERCIST = positron emission tomography response criteria in solid tumours; $P R=$ partial response; $S D=$ stable disease; $W I S=$ wash-in slope; WOS = wash-out slope; $\Delta$ $=$ percentage change between pre and post treatment

\section{Results}

According to the results reported by our previous study ${ }^{9}$, among 13 patients subjected to MRI, 10 showed a significant response while 3 patients were classified in a stable disease (Table 3).
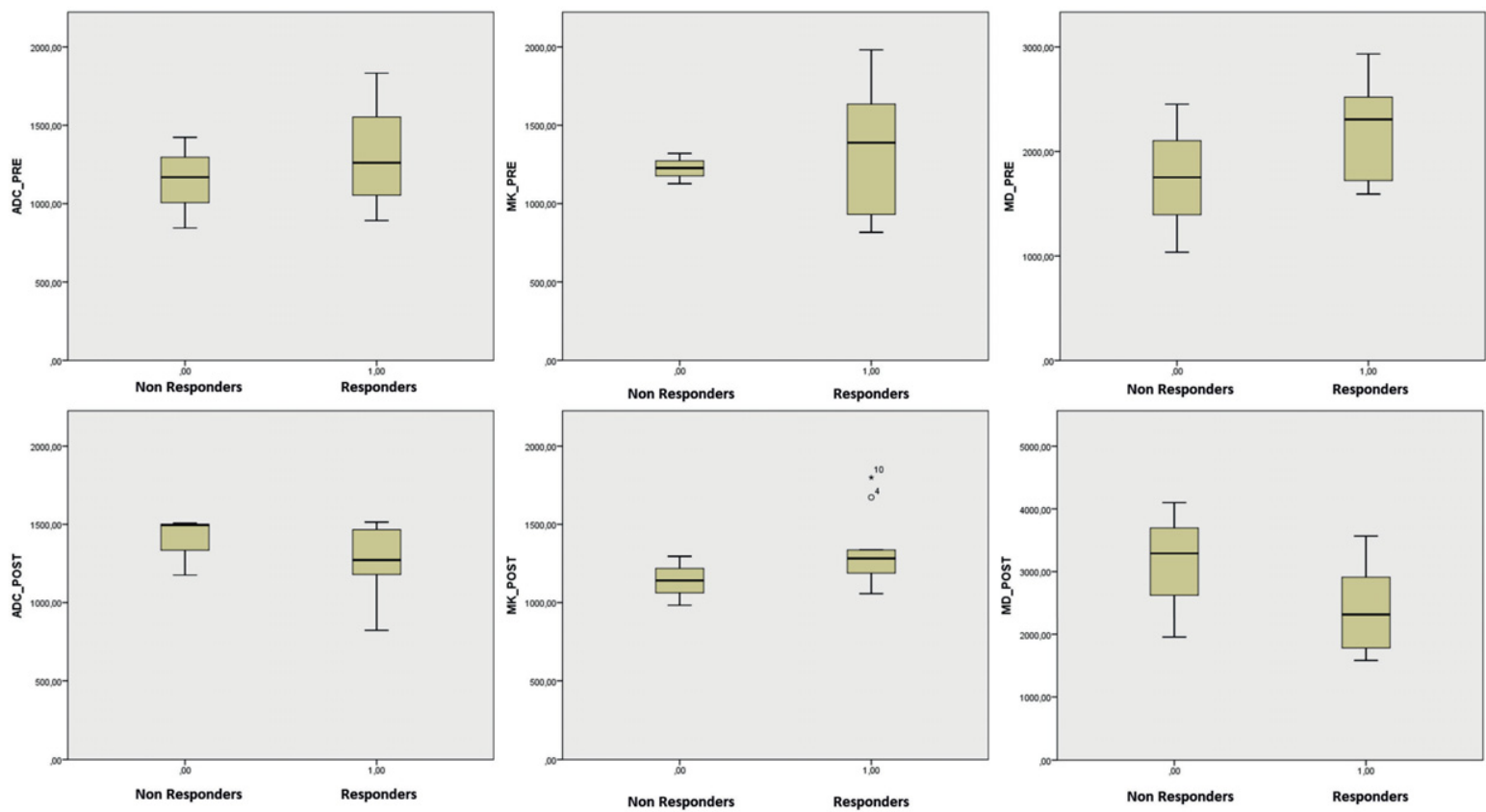

FIGURE 1. Boxplot of apparent diffusion coefficient (ADC) and diffusion kurtosis imaging (DKI) parameters pre and post treatment values between responders and not responders. 

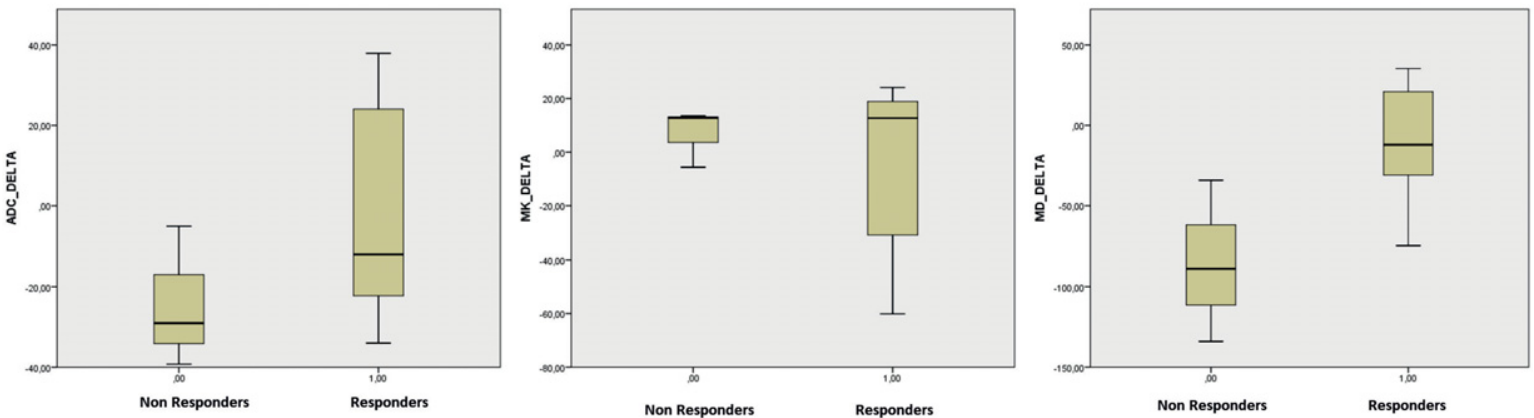

FIGURE 2. Boxplot of apparent diffusion coefficient (ADC) and diffusion kurtosis imaging (DKI) parameters percentage change values between responders and not responders.

Delta $(\Delta)=$ percentage change between pre and post treatment; $M D=$ mean of diffusion coefficient; $M K=$ mean of diffusional kurtosis

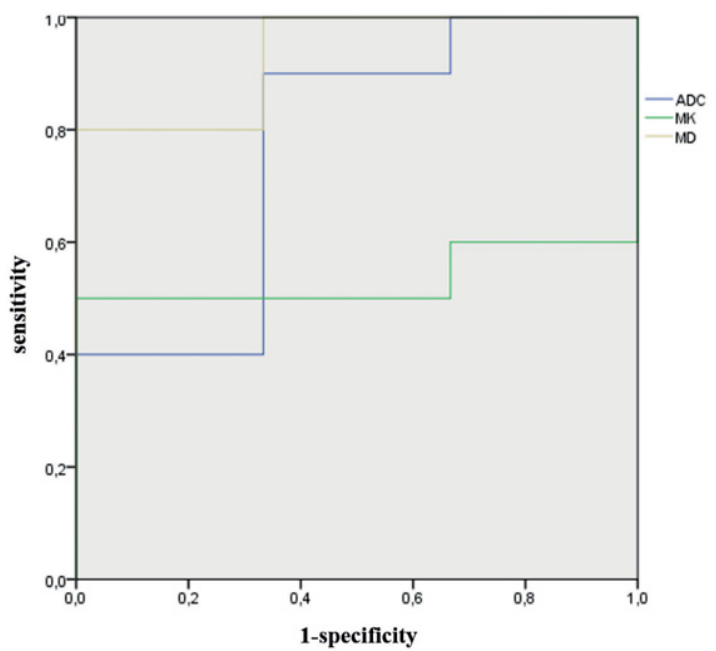

FIGURE 3. ROC curve of apparent diffusion coefficient (ADC) and diffusion kurtosis imaging (DKI) features to assess electrochemotherapy (ECT) response.

$M D=$ mean of diffusion coefficient; $M K=$ mean of diffusional kurtosis

TABLE 4. Diagnostic accuracy of MRI extracted parameters in discrimination of responders and not responders.

\begin{tabular}{ccccccc}
\hline & AUC & $95 \% \mathrm{Cl}$ & p value & Sensitivity & Specificity & Cut-off \\
\hline$\triangle$ ADC & 0,767 & $0,429-1,00$ & 0,176 & 0,900 & 0,667 & $-25,775$ \\
$\Delta$ MK & 0,533 & $0,229-0,837$ & 0,866 & 0,500 & 1,000 & 14,555 \\
$\Delta M D$ & $\mathbf{0 , 9 3 3}$ & $\mathbf{0 , 7 8 2 - 1 , 0 0 0}$ & $\mathbf{0 , 0 2 8}$ & $\mathbf{0 , 8 0 0}$ & 1,000 & $\mathbf{- 3 2 , 5 7 0}$ \\
ADC PRE & 0,667 & $0,292-1,000$ & 0,398 & 0,700 & 0,667 & 1182,550 \\
MK PRE & 0,667 & $0,380-1,953$ & 0,398 & 0,600 & 1,000 & 1348,700 \\
MD PRE & 0,700 & $0,360-1,000$ & 0,310 & 0,400 & 1,000 & 2477,500 \\
ADC POST & 0,367 & $0,000-0,766$ & 0,499 & 0,800 & 0,333 & 1177,825 \\
MK POST & 0,800 & $0,505-1,000$ & 0,128 & 0,500 & 1,000 & 1299,075 \\
MD POST & 0,267 & $0,000-0,602$ & 0,237 & 0,600 & 0,333 & 2020,725 \\
\hline
\end{tabular}

$A D C=$ apparent diffusion coefficient $; C l=$ confidence interval; $M D=$ mean of diffusion coefficient; MK = mean of diffusional kurtosis
ADC and MK did not show differences statistically significant between the values pre and post treatment ( $\mathrm{p}$ value $=0.02$ at Wilcoxon test) and between the percentage changes of responder and not responder patients ( $\mathrm{p}$ value $>0.05$ at Wilcoxon test). Only MD showed a significant variation of values between pre and post treatment ( $\mathrm{p}$ value $=0.02$ at Wilcoxon test) and a significant statistically difference for percentage changes between responders and not responders ( $p$ value $=0.01$ at Kruskal Wallis test).

Figure 1 reports boxplot for each parameter pre and post treatment between responder and not responder patients. Figure 2 shows boxplot for percentage change (delta) between pre and post treatment for each parameters.

Table 4 reports the diagnostic performance of ADC and DKI derived parameters to assess ECT response.

Exclusively MD, among investigated parameters, had a good diagnostic performance with a sensitivity of $80 \%$, a specificity of $100 \%$ (Table 1 ) and area under ROC of 0.933 (Figure 3).

Figure 4 shows a case of significant response on conventional MR images, ADC map and DKI derived parameters maps.

\section{Discussion}

ECT treatment is a very promising tool in oncological patients. The establishment and expansion of ECT in deep-seated tumours (e.g., liver, bone metastases, pancreas) has opened new opportunities for minimally invasive treatment of metastases and carcinomas. ${ }^{7}$ ECT is usually applied as palliative treatment for patients with not resectable lesion, causing an improvement of quality 


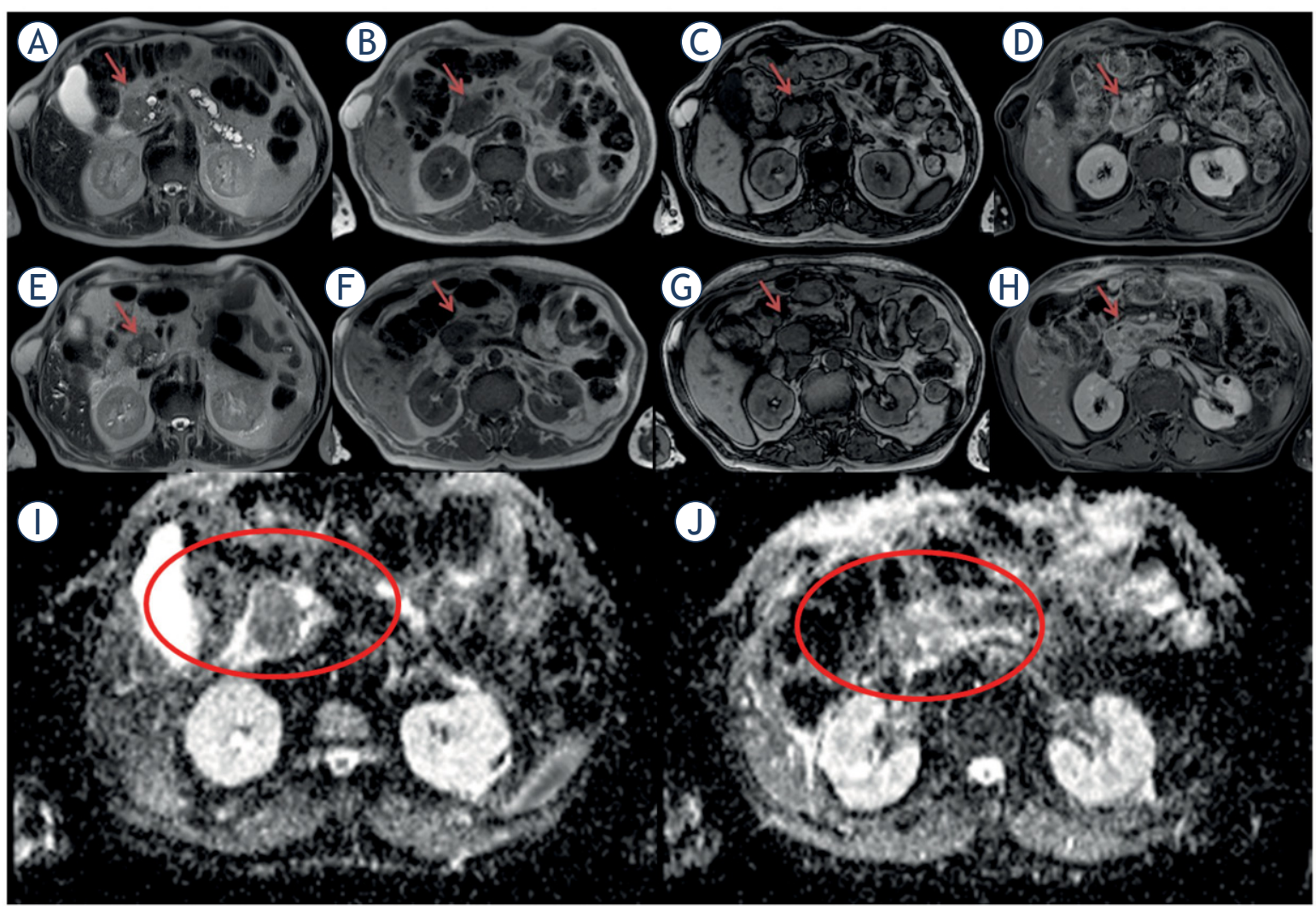

FIGURE 4. Adenocarcinoma of the pancreatic head. Before treatment in (A) (half-Fourier acquisition single-shot turbo spin-echo [HASTE] T2- Weighted [W] sequence), the lesion (arrow) appears hyperintense, in (B) (in-phase T1-W sequence) and (C) (out-phase $\mathrm{Tl}$-W sequence) appears hypointense and hypovascular in (D) (volumetric interpolated breath hold examination [VIBE] TI-W in equilibrium phase). After the treatment the lesion in (E) (HASTE T2-W sequence), (F) (in-phase T1-W sequence), (G) (out-phase TI-W sequence) and (H) (VIBE TI-W in equilibrium phase): there were not significant differences in size and signal compared to baseline. Apparent diffusion coefficient (ADC) map before and after treatment $(\mathrm{I}, \mathrm{J})$.

of life. Several researchers have evaluated it as a treatment of advanced neoplastic lesions in which radical surgery is not possible (e.g., due to lesion location, size, and/or number). ECT allows treatment of lesions in proximity of vital structures like vessels and nerves. The safety profile of ECT is favourable, with local and transient adverse events. ${ }^{7}$ Safety and efficacy of ECT have been showed in a prospective series of patients with unresectable Perihilar-Cholangiocarcinoma (PHCCA). ${ }^{5}$ A prospective study conducted in 10 patients suffering from hepatocellular carcinomas (HCC) proved that ECT is a feasible and safe treatment. At average of 20.5 months after ECT 15 out of 17 lesions showed a complete response. ${ }^{27}$ Recently, Boc et al. characterized ultra-sonographic (US) findings during and after electrochemotherapy of liver. US provides a tool for assessment of appropriate electrode insertion for intraoperative electrochemotherapy of liv- er tumours and assessment of the appropriate coverage of a tumour with a sufficiently strong electric field and can serve as predictor of the response of tumours. ${ }^{28}$ As we reported in our previous studies, ECT can be used in locally advanced pancreatic cancer with no side effects or major complications to surrounding viscera that required medical or surgical treatment. ${ }^{6-8}$ The major limit of our study is due to how we should assess the ablated area. In fact, we demonstrated in ${ }^{9}$ that morphological assessment based on RECIST criteria in not sufficient to stratify the patients as responders or not responders. ${ }^{9}$ Conversely, a functional assessment, based on the evaluation of parameters extracted by DCE-MRI and DWI, could be used to identify responders and not responders patients. ${ }^{9}$ In the current study, we evaluated conventional DWI parameters $(\mathrm{ADC})$ and the parameters extracted by DKI (MD and MK) in the assessment of ECT 
treatment. Only MD by DKI showed a significant variation of value between pre and post treatment and a significant statistically difference for percentage change between responders and not. When we evaluated the diagnostic accuracy of ADC and DKI parameters, MD had a good accuracy with and area under ROC of 0.933 . To the best of our knowledge, the few reports on Kurtosis in the assessment of pancreatic tumours, investigate the role of these functional parameters during the characterization of the lesions ${ }^{29}$, other reports on the post treatment assessment by DKI, are not related to pancreas tumours. ${ }^{30-33}$

Diffusion is related to the random Brownian motion of water molecules. In a homogenous medium, diffusion is described by a Gaussian model, while in living tissues is related also by interactions with other molecules and cell membranes and is therefore thought to follow a non-Gaussian model. Also, ADC, in conventional DWI, is influenced by b-values acquired. ${ }^{10}$ DKI is an extension of diffusion tensor imaging that evaluates the microstructure features of tissues in a non-Gaussian model. ${ }^{19}$ Two quantitative parameters can be extracted by DKI model, including kurtosis values $(K$, representing deviation from a Gaussian distribution) and diffusion coefficient $(\mathrm{D}$, defining as a corrected ADC for non-Gaussian bias). ${ }^{19}$ According to Jensen et al. DKI should exhibit a higher sensitivity than conventional DWI for cancer detection. ${ }^{19}$ Shen et al. assessed the accuracy of DKI parameters in evaluating malignant from benign lesions, performing a systematic meta-analysis. ${ }^{34}$ They evaluated 14 studies from 2012 to 2018, for a total of 1847 lesions (895 benign and 952 malignant) in 1107 patients; only two studies were performed with a 1.5 Tesla MR system. The diagnostic performance of three quantitative data, K, D and ADC obtained from MRI with DKI and DWI, for differentiating malignant from benign lesions was evaluated. Pooled results indicated both $\mathrm{K}$ and $\mathrm{D}$ had a good or excellent diagnostic performance in separating malignant cancer from benign lesions, but D may be more superior because it had the higher AUC. ${ }^{30}$ No study on pancreatic cancer was included in this meta-analysis.

Hu et al. evaluated DKI in assessing response in rectal cancer compared to conventional DWI. ${ }^{33}$ They evaluated ADC, MD (mean diffusion), and MK (mean kurtosis) showing that the $\mathrm{MK}_{\text {pre }}$ and $\mathrm{MK}_{\text {post }}$ were much lower for the responder than for not responder patients. Also, the $\mathrm{MD}_{\text {post }}$ and the change ratio of MD increased, whereas there are no significant differences for $\mathrm{ADC}_{\mathrm{pre}} \mathrm{MD}_{\mathrm{pre}}$ and the change of MK ratio between responder and not responder patients. The $\mathrm{MK}_{\text {post }}$ had higher sensitivity and specificity compared to other data. ${ }^{33}$

DWI has demonstrated to be a great promise as an imaging biomarker. However, it still suffers from inconsistencies in imaging acquisition parameters and DWI analysis that lead to substantial issues with reproducibility. Newer imaging techniques such as DKI may extend the potential of DWI by better characterizing and assessing the lesions pre and after treatment. Therefore, DKI should be added to the routine protocol. Several limitations in our study must be mentioned. First, since this was a retrospective study, there may have been potential selection bias. It will be necessary to perform larger studies to validate our findings. Second our data were acquired with a maximum $b$ value of $1000 \mathrm{~s} / \mathrm{mm}^{2}$. In general, very high b-values are recommended for the evaluation of non-Gaussian kurtosis in brain applications. ${ }^{19}$ However, for abdominal studies, taking into account the lower signal to noise ratio (SNR) and the lower T2 relaxation times of the various organs compared to the brain, very high b-values are not usually applied. Recently, various authors have shown that kurtosis effects could be detectable in abdominal and whole-body applications even when using maximum b-values of $800 \mathrm{~s} / \mathrm{mm}^{2}$ or less at 3T.35-37 We applied multiple b-values with a maximum of $1000 \mathrm{~s} / \mathrm{mm}^{2}$ that, coupled with the use of a parallel imaging factor, resulted in images with acceptable SNR at 1.5T. Third, the size of our population is very small. In fact, this study had the only objective to report preliminary findings that should be validate in a larger series of patients. At the end, we did not assess the quality of DWI images; this could be considered a future endpoint.

\section{Conclusions}

Our results support the hypothesis that Mean of Diffusion Coefficient derived by DKI allows to identify responders and not responder patients subject to ECT treatment. MD had high diagnostic performance to assess ECT response, showing a significant variation of value between pre and post treatment and a significant statistically difference for percentage change between responders and not responders patients. 


\section{Acknowledgements}

The authors are grateful to Alessandra Trocino, librarian at the National Cancer Institute of Naples, Italy. Additionally, authors are grateful to Rita Guarino and Assunta Zazzaro for their collaboration.

\section{References}

1. Tarantino L, Busto G, Nasto A, Fristachi R, Cacace L, Talamo M, et al. Percutaneous electrochemotherapy in the treatment of portal vein tumor thrombosis at hepatic hilum in patients with hepatocellular carcinoma in cirrhosis: a feasibility study. World J Gastroenterol 2017; 23: 906-18. doi: 10.3748/wjg.v23.i5.906

2. Edhemovic I, Gadzijev E, Brecelj E, Miklavcic D, Kos B, Zupanic A, et al. Electrochemotherapy: a new technological approach in treatment of metastases in the liver. Technol Cancer Res Treat 2011; 10: 475-85. doi: 10.7785/ tcrt.2012.500224

3. Miklavcic D, Snoj M, Zupanic A, Kos B, Cemazar M, Kropivnik M, et al. Towards treatment planning and treatment of deep-seated solid tumors by electrochemotherapy. Biomed Eng Online 2010; 9: 10. doi: 10.1186/1475925X-9-10

4. Tafuto S, von Arx C, De Divitiis C, Maura CT, Palaia R, Albino V, et al.; ENETS Center of Excellence Multidisciplinary Group for Neuroendocrine Tumors in Naples (Italy). Electrochemotherapy as a new approach on pancreatic cancer and on liver metastases. Int J Surg 2015; 21(Suppl 1): S78-82. doi: 10.1016/j.ijsu.2015.04.095

5. Tarantino L, Busto G, Nasto A, Nasto RA, Tarantino P, Fristachi R, et al. Electrochemotherapy of cholangiocellular carcinoma at hepatic hilum: a feasibility study. Eur J Surg Oncol 2018; pii: S0748-7983(18) 31169-7. doi: 10.1016/j.ejso.2018.06.025

6. Granata V, Fusco R, Piccirillo M, Palaia R, Lastoria S, Petrillo A, et al. Feasibility and safety of intraoperative electrochemotherapy in locally advanced pancreatic tumor: a preliminary experience. Eur J Inflamm 2014; 12: 467-77. doi: 10.1177/1721727X1401200308

7. Probst U, Fuhrmann I, Beyer L, Wiggermann P. Electrochemotherapy as a new modality in interventional oncology: a review. Technol Cancer Res Treat 2018; 17: 1533033818785329. doi: 10.1177/1533033818785329

8. Granata V, Fusco R, Piccirillo M, Palaia R, Petrillo A, Lastoria S, et al. Electrochemotherapy in locally advanced pancreatic cancer: preliminary results. Int J Surg 2015; 18: 230-6. doi: 10.1016/j.jjsu.2015.04.055

9. Granata V, Fusco R, Setola S, Piccirillo M, Leongito M, Palaia R, et al. Early radiological assessment of locally advanced pancreatic cancer treated with electrochemotherapy. World J Gastroenterol 2017; 23: 4767-78. doi: 10.3748/wjg.v23.i26.4767

10. García-Figueiras R, Padhani AR, Baleato-González S. Therapy monitoring with functional and molecular MR imaging. Magn Reson Imaging Clin N Am 2016; 24: 261-288. doi: 10.1016/j.mric.2015.08.003

11. Fusco R, Sansone M, Petrillo A. A comparison of fitting algorithms for diffusion-weighted MRI data analysis using an intravoxel incoherent motion model. MAGMA 2017; 30: 113-120. doi: 10.1007/s10334-016-0591-y

12. Petrillo M, Fusco R, Catalano O, Sansone M, Avallone A, Delrio P, et al. MRI for assessing response to neoadjuvant therapy in locally advanced rectal cancer using DCE-MR and DW-MR data sets: a preliminary report. Biomed Res Int 2015; 2015: 514740, doi: 10.1155/2015/514740

13. Doblas S, Wagner M, Leitao HS, Daire JL, Sinkus R, Vilgrain V, et al. Determination of malignancy and characterization of hepatic tumor type with diffusion-weighted magnetic resonance imaging: comparison of apparent diffusion coefficient and intravoxel incoherent motion-derived measurements. Invest Radiol 2013; 48: 722-8. doi: 10.1097/RLI.0b013e3182915912

14. Concia M, Sprinkart AM, Penner AH, Brossart P, Gieseke J, Schild HH, et al. Diffusion-weighted magnetic resonance imaging of the pancreas: diagnostic benefit from an intravoxel incoherent motion model-based $3 \mathrm{~b}$-value analysis. Invest Radiol 2014; 49: 93-100. doi: 10.1097/RLI.0b013e3182a71cc3
15. Chandarana H, Kang SK, Wong S, Rusinek H, Zhang JL, Arizono S, et al. Diffusion-weighted Intravoxel incoherent motion imaging of renal tumors with histopathologic correlation. Invest Radiol 2012; 47: 688-96. doi: 10.1097/RLI.0b013e31826a0a49

16. Kobus T, Vos PC, Hambrock T, De Rooij M, Hulsbergen-Van de Kaa CA, Barentsz JO, et al. Prostate cancer aggressiveness: in vivo assessment of MR spectroscopy and diffusion-weighted imaging at 3 T. Radiology 2012; 265: 457-67. doi: 10.1148/radiol.12111744

17. Le Bihan D, Breton E, Lallemand D, Aubin ML, Vignaud J, Laval-Jeantet $M$. Separation of diffusion and perfusion in intravoxel incoherent motion MR imaging. Radiology 1988; 168: 497-505. doi: 10.1148/radiology.168.2.3393671

18. Le Bihan D, Breton E, Lallemand D, Grenier P, Cabanis E, Laval-Jeantet M. MR imaging of intravoxel incoherent motions: application to diffusion and perfusion in neurologic disorders. Radiology 1986; 161: 401-7. doi: 10.1148/ radiology.161.2.3763909

19. Jensen $\mathrm{JH}$, Helpern JA. MRI quantification of non-Gaussian water diffusion by kurtosis analysis. NMR Biomed 2010; 23: 698-710. doi: 10.1002/ nbm.1518

20. Sun K, Chen X, Chai W, Fei X, Fu C, Yan X, et al. Breast cancer: diffusion kurtosis MR imaging-diagnostic accuracy and correlation with clinical-pathologic factors. Radiology 2015; 277: 46-55. doi: 10.1148/radiol.15141625

21. Suo S, Chen X, Wu L, Zhang X, Yao Q, Fan Y, et al. Non-Gaussian water diffusion kurtosis imaging of prostate cancer. Magn Reson Imaging 2014; 32: 421-27. doi: 10.1016/j.mri.2014.01.015

22. Nogueira L, Brandão S, Matos E, Nunes RG, Loureiro J, Ramos I, et al. Application of the diffusion kurtosis model for the study of breast lesions. Eur Radiol 2014; 24: 1197-203. doi: 10.1007/s00330-014-3146-5

23. Rosenkrantz AB, Sigmund EE, Winnick A, Niver BE, Spieler B, Morgan GR, et al. Assessment of hepatocellular carcinoma using apparent diffusion coefficient and diffusion kurtosis indices: preliminary experience in fresh liver explants. Magn Reson Imaging 2012; 30: 1534-40. doi: 10.1016/j. mri.2012.04.020

24. Kartalis N, Manikis GC, Loizou L, Albiin N, Zöllner FG, Del Chiaro M, et al. Diffusion-weighted MR imaging of pancreatic cancer: a comparison of mono-exponential, bi-exponential and non-Gaussian kurtosis models. Eur Radiol Open 2016; 3: 79-85. doi: 10.1016/j.ejro.2016.04.002

25. Weng Z, Ertle J, Zheng S, Lauenstein T, Mueller S, Bockisch A, et al. Cho criteria are superior in evaluating tumor response in patients treated with transarterial radioembolization for hepatocellular carcinoma. Oncol Lett 2013; 6: 1707-12. doi: 10.3892/ol.2013.1612

26. Wahl RL, Jacene $H$, Kasamon $Y$, Lodge MA. From RECIST to PERCIST: evolving considerations for PET response criteria in solid tumors. J Nucl Med 2009; 50 (Suppl 1): 122S-150S. doi: 10.2967/jnumed.108.057307

27. Djokic M, Cemazar M, Popovic P, Kos B, Dezman R, Bosnjak M, et al. Electrochemotherapy as treatment option for hepatocellular carcinoma, a prospective pilot study. Eur J Surg Oncol 2018; 44: 651-57. doi:10.1016/j. ejso.2018.01.090

28. Boc N, Edhemovic I, Kos B, Music MM, Brecelj E, Trotovsek B, et al. Ultrasonographic changes in the liver tumors as indicators of adequate tumor coverage with electric field for effective electrochemotherapy. Radiol Oncol 2019; 53(1): 15-24.; 53(1): 15-24.; 52: 383-91. doi: 10.2478/ raon-2018-0041

29. Tang MY, Zhang XM, Chen TW, Huang XH. Various diffusion magnetic resonance imaging techniques for pancreatic cancer. World J Radiol 2015; 7: 424-37. doi:10.4329/wjr.v7.i12.424

30. Chen Y, Ren W, Zheng D, Zhong J, Liu X, Yue Q, et al. Diffusion kurtosis imaging predicts neoadjuvant chemotherapy responses within 4 days in advanced nasopharyngeal carcinoma patients. J Magn Reson Imaging 2015 42: 1354-61. doi: 10.1002/jmri.24910.

31. Yu J, Xu Q, Song JC, Li Y, Dai X, Huang DY, et al. The value of diffusion kurtosis magnetic resonance imaging for assessing treatment response of neoadjuvant chemoradiotherapy in locally advanced rectal cancer. Eur Radiol 2017; 27: 1848-57. doi: 10.1007/s00330-016-4529-6

32. Goshima S, Kanematsu M, Noda Y, Kondo H, Watanabe H, Bae KT. Diffusion kurtosis imaging to assess response to treatment in hypervascular hepatocellular carcinoma. AJR Am J Roentgenol 2015; 204: W543-9. doi: 10.2214/ AJR.14.13235 
33. Hu F, Tang W, Sun Y, Wan D, Cai S, Zhang Z, et al. The value of diffusion kurtosis imaging in assessing pathological complete response to neoadjuvant chemoradiation therapy in rectal cancer: a comparison with conventional diffusion-weighted imaging. Oncotarget 2017; 8: 75597-606. doi: 10.18632/ oncotarget.17491

34. Shen L, Zhou G, Tang F, Lin Y, Zhou J, Lv P, et al. MR diffusion kurtosis imaging for cancer diagnosis: a meta-analysis of the diagnostic accuracy of quantitative kurtosis value and diffusion coefficient. Clin Imaging 2018; 52: 44-56. doi: 10.1016/j.clinimag.2018.06.005

35. Kartalis N, Lindholm TL, Aspelin P, Permert J, Albiin N. Diffusion-weighted magnetic resonance imaging of pancreas tumors. Eur Radiol 2009; 19: 1981-90. doi: 10.1007/s00330-009-1384-8

36. Fusco R, Sansone M, Granata V, Grimm R, Pace U, Delrio P, et al. Diffusion and perfusion MR parameters to assess preoperative short-course radiotherapy response in locally advanced rectal cancer: a comparative explorative study among Standardized Index of Shape by DCE-MRI, intravoxel incoherent motion- and diffusion kurtosis imaging-derived parameters. Abdom Radiol (NY). 2018 Oct 25.

37. Rosenkrantz AB, Sigmund EE, Johnson G, Babb JS, Mussi TC, Melamed J, et l. Prostate cancer: feasibility and preliminary experience of a diffusional kurtosis model for detection and assessment of aggressiveness of peripheral zone cancer. Radiology 2012; 264: 126-35. doi: 10.1148/radiol.12112290 